\title{
Power Harvesting from Microwave Oven Electromagnetic Leakage
}

\author{
Yoshihiro Kawahara*† \\ kawahara@akg.t.u-tokyo.ac.jp \\ Rushi Vyas ${ }^{\dagger}$ \\ rushi.vyas@gatech.edu
}

\author{
Xiaoying Bian* \\ bian@akg.t.u-tokyo.ac.jp \\ Manos M. Tentzeris ${ }^{\dagger}$ \\ etentze@ece.gatech.edu
}

\author{
Ryo Shigeta* \\ shigeta@akg.t.u-tokyo.ac.jp \\ Tohru Asami* \\ asami@akg.t.u-tokyo.ac.jp
}

\author{
*The University of Tokyo \\ 7-3-1 Hongo, Bunkyo-ku \\ Tokyo, 113-8656, Japan
}

${ }^{\dagger}$ Georgia Institute of Technology 85 5th Street NW

Atlanta, GA 30308, U.S.A.

\begin{abstract}
In this paper, we considered the possibility of using electricity harvested from the microwave field leaked from commercial microwave ovens. Our experimental results showed that the leakage received by a dipole antenna was about $0 \mathrm{dBm}$ $(1 \mathrm{~mW})$ at a point $5 \mathrm{~cm}$ in front of the door. A rectenna consisting of a dipole antenna and charge pump can convert the leaked microwave energy into a DC current. When a microwave oven is operated for $2 \mathrm{~min}, 9.98 \mathrm{~mJ}$ of energy was harvested. We demonstrated that this energy is sufficient for powering a digital cooking timer to count down for $3 \mathrm{~min}$ and beep for $2.5 \mathrm{~s}$. The operation of other kitchen devices was also demonstrated.
\end{abstract}

\section{Author Keywords}

Energy harvesting; microwave oven; wireless sensing.

\section{ACM Classification Keywords}

B.m. Hardware: Miscellaneous

\section{General Terms \\ Design; Measurement}

\section{INTRODUCTION}

Power supply is always an issue for the perpetual operation of electronics devices. Low-power electrical devices used at home, such as wristwatches, toys, and kitchen tools, are powered by button cell batteries, which act as a disposable power source. However, button cells are used because they are convenient for the manufacturer rather than benefit the end users. The capacity of button cells is limited to several hundred milliamp-hours depending on the electrochemical system used. Although button cells are intended for use in low-power consuming devices with long service lives, the users still need to replace them regularly. Hundreds of standard cells exist. Users often find it difficult to choose the

Permission to make digital or hard copies of all or part of this work for personal or classroom use is granted without fee provided that copies are not made or distributed for profit or commercial advantage and that copies bear this notice and the full citation on the first page. Copyrights for components of this work owned by others than ACM must be honored. Abstracting with credit is permitted. To copy otherwise, or republish, to post on servers or to redistribute to lists, requires prior specific permission and/or a fee. Request permissions from permissions@acm.org.

UbiComp'13, September 8-12, 2013, Zurich, Switzerland.

Copyright (c) 2013 ACM 978-1-4503-1770-2/13/09...\$15.00

http://dx.doi.org/10.1145/2493432.2493500

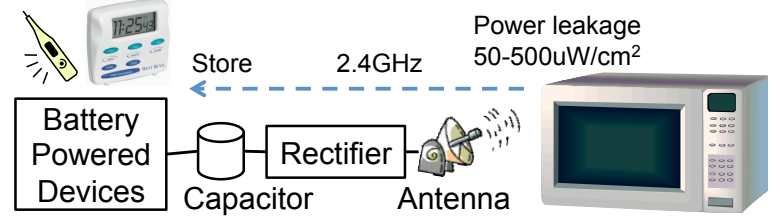

Figure 1. Concept of energy harvesting from microwave oven leakage.

right one at the retail store. Moreover, even though mercuric oxide button cells are no longer used, some batteries still contain small amount of mercury for anti-corrosion. Therefore, battery recycling associations suggest for users to recycle the used button cells, which also increases the end-user's time and effort spent.

Thanks to the reduced power consumption of electronics devices, quite a few battery operated devices such as kitchen tools only consumes a few dozen microwatts. This reduced power requirements are accelerating power harvesting from various ambient energy. For instance, power consumption of a cooking thermometer (Tanita TT-533-GR), humidity and temperature meter (Dretec O-226 WT) are 23.9 and $70 \mu \mathrm{W}$, respectively. Off-the-shelf wireless sensing platform such as Texas Instruments eZ430-RF2500 sensor can sense temperature data and send it to a remote sink node using only $200 \mu \mathrm{J}$ and current consumption in sleep mode is about $1 \mu \mathrm{A}$ [13]. As energy efficiency continues to improve, the energy requirements to power electronic devices will continue to drop; this in turn means it is feasible to power more devices by a small amount of energy of about a few dozen microwatts.

In this paper, we present the feasibility of harvesting and storing a small amount of leaked energy from a microwave oven and operate low-power devices without battery in a domestic environment. As shown in Figure 1, a combination of antenna and rectifier can be used to collect a few hundreds of microwatts of energy from the leakage electromagnetic field of a microwave oven and store the energy in a capacitor. By accumulating this energy over time, we can use it to provide power to low-power devices.

There are several advantages to harvest leakage from microwave ovens: (1) most households own a microwave oven; (2) leakage power from a microwave oven is much stronger than any other sources at home; (3) the leakage power is 
wasted, and harvesting the leaked power does not affect heating operation of the oven; (4) a power harvesting device, called a rectenna, can be manufactured at extremely low cost; and (5) microwave ovens are usually located in kitchens with many battery-operated tools, such as a weight scale, a timer, and thermometers.

We note that harvesting small amounts of energy from ambient radio waves is not a new idea. Recently, WARP [9, $11]$ and other ambient energy harvesting wireless sensor networks $[8,14]$ have proposed power harvesting from broadcasting TV towers. The leakage from a microwave oven is also well known, especially from the perspective of electromagnetic compatibility [7]. To the best of our knowledge, however, there have been no reports presenting system-level quantitative characterization results on how much energy can be harvested from microwave oven leakage.

Our contributions are summarized as follows. We extensively measured the leakage level from microwave ovens in terms of the variation for models and manufacturers, frequency, operation time, and contents. In addition, we fabricated an energy harvesting circuit rectenna specially tuned to the power level and frequency of the microwave oven. We demonstrated that the power harvested by the rectenna can actually be stored in a capacitor and reused to power a wide variety of low-power devices such as thermometers, digital weight scales, and gas sensors. Through the measurement, we also found the variation of leaked energy depends on the contents and status of the contents in the microwave oven chamber. This result suggests the possibility of inferring the cooking state out of the microwave oven.

\section{RELATED WORKS}

Energy harvesting is being actively researched in the hope of realizing energetically autonomous wireless sensor networks. Most wireless sensor network platforms come with an MCU, flash memory, RF transceivers, and an A/D converter for sensors. These components typically consume several dozen milliwatts for operation. The nodes form an ad-hoc network and deliver the data. Most existing "energy harvesting enabled" wireless sensor network platforms use this architecture $[8,9]$ and consume a few tens of milliwatts. When sufficient energy cannot be scavenged from the ambient environment, a node needs to remain dormant for a while.

The use of radio waves as a means to transfer electricity was attempted by Nicola Tesla in the early twentieth century. This far-field wireless power transmission technique is, in principle, similar to ambient RF energy harvesting. Passive RFID[6, 10] and space-based solar power (SBSP) [2] are also categorized as far-field wireless power transmission techniques. In passive RFID systems, a transponder (RFID card) is only activated when it is within the interrogation zone of a reader. The power required to activate the transponder is supplied to the transponder through the coupling unit. Long range RFID systems operate at UHF frequencies of $915 \mathrm{MHz}$, 2.5 and $5.8 \mathrm{GHz}$. Since transponder chips consumes no more than $5 \mu \mathrm{W}$ and the transponder is designed to be powered by the interrogator, there are fewer challenges in terms of power

\begin{tabular}{|c|c|c|c|}
\hline & Maximum Value & Frequency & Make, Model \\
\hline \hline $\mathrm{A}$ & $295.5 \mu \mathrm{W} / \mathrm{cm}^{2}$ & $2465 \mathrm{MHz}$ & Whirlpool, TMH16XSD-2 \\
B & $6194.7 \mu \mathrm{W} / \mathrm{cm}^{2}$ & $2472 \mathrm{MHz}$ & Haier, MWM11100TW \\
C & $246.8 \mu \mathrm{W} / \mathrm{cm}^{2}$ & $2445 \mathrm{MHz}$ & Sharp, R55TS \\
D & $400.7 \mu \mathrm{W} / \mathrm{cm}^{2}$ & $2445 \mathrm{MHz}$ & Panasonic, NN-S446BA \\
E & $363.6 \mu \mathrm{W} / \mathrm{cm}^{2}$ & $2457.5 \mathrm{MHz}$ & Kenmore 721.8911980 \\
F & $206.1 \mu \mathrm{W} / \mathrm{cm}^{2}$ & $2465.5 \mathrm{MHz}$ & National, NE-EZ2 \\
\hline
\end{tabular}

Table 1. Maximum leakage level of microwave ovens.

level and range compared to harvesting from microwave leakage.

A highly efficient rectifier and antenna (rectenna) were intensively developed for SPSS and so on $[4,5]$. SPSS is a concept of generating electricity in space using photovoltaics and delivering the power to the ground by microwaves. However, the RF power density and rectenna operating point remain virtually constant with time; they require minimal power management circuitry to extract peak power.

WISP is a promising platform that employs battery-free sensing [12]. WISP is compliant with the EPC Class 1 Generation 1 protocol - i.e., WISP sensor tags harvest energy from a standard RFID reader and reflect the reader's signal to transmit the sensed data. This approach inherently requires an RFID reader, which often requires a constant power supply. The experimental results showed that an antenna $(5 \mathrm{dBi})$ designed for TV applications and four-stage power harvesting circuit can be used to scavenge $60 \mu \mathrm{W}$ of power [11].

PL-Tags are battery-less tags that can be excited wirelessly by using an existing phenomenon from power lines. It exploits electrical transient pulses that result from the switching of electrical loads over a power line [10]. This approach is unique in that it requires very little additional infrastructure. However, electromagnetic noise such as transient pulses does not travel very far through air. Thus, the effective distance between a tag and power line is limited to several meters.

\section{MICROWAVE OVEN AND ITS LEAKAGE}

In this section, we review mechanism, regulation, and actual leakage power of microwave oven that are important for designing a power harvester. A microwave oven is a kitchen appliance that heats foods using electromagnetic radiation in the microwave spectrum. Dielectric substances such as water contained in food are rotated by a high-frequency alternating electric field, and friction heat is generated as a result of the rotation. Most microwave ovens operate at $2.4 \mathrm{GHz}$, which is one of the industrial, scientific, and medical (ISM) radio bands.

The Center for Devices and Radiological Health (CDRH) of the United States Food and Drug Administration (FDA) defines performance standards for microwave ovens with regard to the leakage level and safety features. According to Title 21, CFR, Part 1030[1], the power density in the proximity of the external oven surface shall not exceed $1 \mathrm{~mW} / \mathrm{cm}^{2}$ at any point $5 \mathrm{~cm}$ or more from the external surface of the oven measured prior to acquisition by a purchaser and, thereafter, 5 $\mathrm{mW} / \mathrm{cm}^{2}$ at any such point. We measured several home microwave ovens. Narda SRM-3000 was used to measure the 


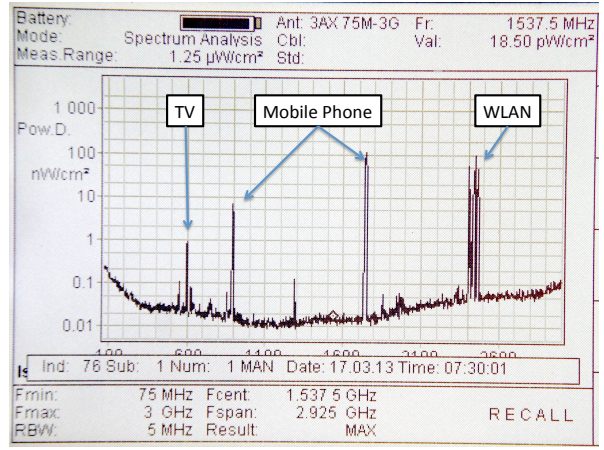

Figure 2. Maximum power density of indoor environment (75 MHz-3 GHz).

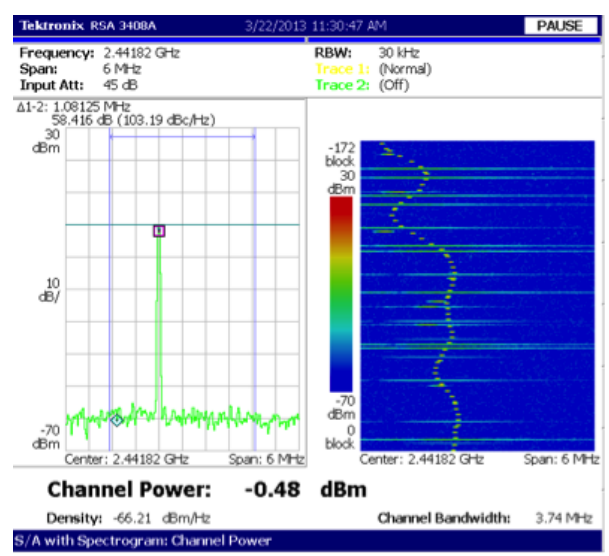

Figure 3. Spectrogram of power leakage from microwave oven.

maximum power density radiated when $275 \mathrm{~mL}$ of tap water was heated in the microwave oven for $30 \mathrm{~s}$ as specified by $[1]^{1}$. The measurement results in Table 1 show the leakage levels are below the regulation limit.

Figure 2 shows the measured maximum power density in an indoor environment. The measurement was taken at a university laboratory. We could observe the power density of some outstanding communication and broadcasting systems. However, all of the power densities were below $100 \mu \mathrm{W} / \mathrm{cm}^{2}$, which is 100-1,000 times smaller than the leaked power from the microwave ovens. The maximum leakage power was always measured at the front door panel for all of the target microwave ovens.

In terms of power harvesting, average power density is also important measure to estimate the feasibility. However it turned out that measurement of absolute value was difficult because the frequency and value of the strongest power frequently changes. Figure 3 shows the measured spectrogram when the microwave oven NE-EZ2 was in operation ${ }^{2}$. As shown in Figure 3, the peak frequency changed slightly over

\footnotetext{
${ }^{1}$ A number of inexpensive "home" microwave meters may not be accurate enough for survey work; these inexpensive meters usually cannot be calibrated to meet the standards.

${ }^{2}$ A dipole antenna fabricated on a printed circuit board was connected to a spectrum analyzer Tektronics RSA-3408B by a coaxial cable. The monopole antenna was positioned $5 \mathrm{~cm}$ away from the door of the oven.
}

time. This is very different phenomenon compared to what you see in communication and broadcasting signal. Moreover, as seen in the spectrogram in Figure 3, the maximum value is not continuous in time domain too. The spectrum analyzer indicates the channel power is $-0.48 \mathrm{dBm}(0.9 \mathrm{~mW})$. We would note that this value is instantaneous value this screenshot was captured rather than a constant value.

\section{DESIGN AND IMPLEMENTATION OF RECTENNA}

In the introduction, we noted that a rectenna can be manufactured at extremely low cost. This is because the simplest rectenna consists of low cost components such as an antenna, a diode, and a capacitor. The size of the harvester is small enough that it could be integrated inside most devices. Even though the manufacturing cost is low, the design and implementation of a circuit for harvesting power from microwave power still require higher engineering skills. One reason is that the power level of a microwave oven is much lower than that of wireless power transmission. The incoming power transduced across the antenna is in RF form and induces a very low voltage across the antenna terminals. Harnessing this power requires it to be rectified and stepped up to an output voltage of $1.8 \mathrm{~V}$ or higher, at which most electronic devices such as embedded processors and sensors can operate.

\section{Rectifier Design}

The Dickson charge pump is a voltage multiplier widely used for ambient energy harvesting applications. It converts $\mathrm{AC}$ electrical power from a lower voltage to a higher DC voltage using a network of capacitors and diodes. There are a number of considerations needed to design the best charge pump. Using an ideal four-stage Dickson charge pump $(5 \times$ multiplier), an input voltage of $1.0 \mathrm{~V}$ will generate an output of 5.0 $\mathrm{V}$. However, a number of factors reduce the output voltage (e.g., threshold voltage, forward voltage drop of diode, parasitic capacitance to ground). The input power level and load affect the conversion efficiency.

An alternative approach to multiplying power while maintaining high conversion efficiency is the use of a DC-DC integrated charge pump. Recently, Parks et al. demonstrated a combination of a simpler rectifier and low-power DC-DC converter (Seiko S-882Z IC)[9]. We believe this approach will provide practical power management for duty cycle controlled devices such as sensor networks.

The objective of our study was to characterize the power leakage from a microwave oven and show the feasibility of using it as a substitute for button cell batteries. Therefore, we fabricated a Dickson charge pump as a first step toward this goal. The RF charge pump circuit was designed and optimized using the LT-SPICE simulator and optimized for operation in the $2.46 \mathrm{GHz}$ frequency band. The circuit schematic is shown in Figure 4. The topology comprised a five-stage charge pump configuration as this offered the best tradeoff between the voltage gain and loss for non-IC based designs using discrete components onboard that contain higher parasitic losses. Another design using an eight-stage RF charge pump topology suffers 2.5 times as much loss as the five-stage pump[3]. 


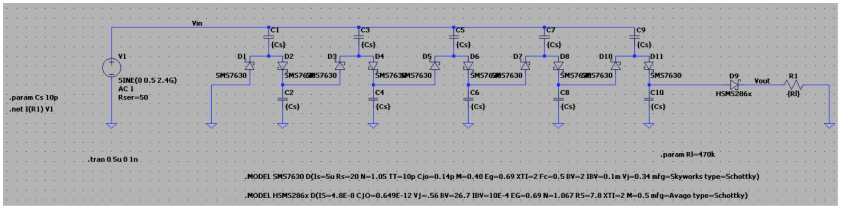

Figure 4. Schematics of a charge pump.

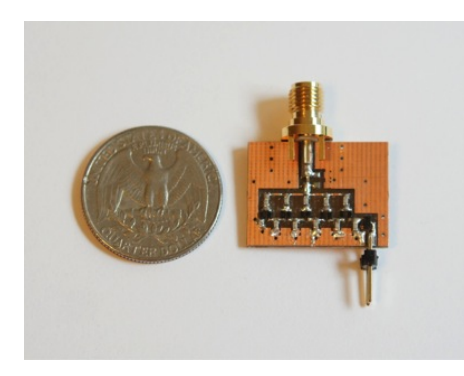

Figure 5. 2.4 GHz rectifier fabricated on FRP4 board (Type A).

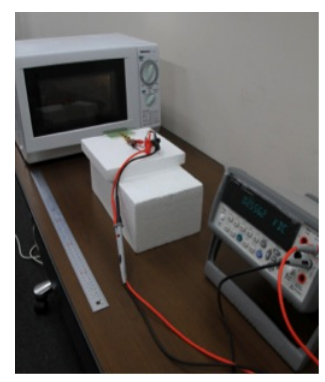

Figure 6. Measurement setup.
We designed two slightly different rectifiers with different optimal load sizes. Type A was designed so that the conversion efficiency would reach its maximum at a load resistance value of about $750 \Omega$ (Figure 5). Type B was optimized for a load resistance value of about $10 \mathrm{k} \Omega$. The topologies of both charge pumps were based on Dickson charge pump. The difference was the choice of diode.

\section{Antenna}

Choice of correct antennas type will also increase the amount of power harvested. The antenna converts the incident electric field in the air to an electrical RF sinusoidal signal. Antennas come in many different shapes and sizes. Given two antennas, the ratio of power available at the input of the receiving antenna $P_{r}$ to output power to the transmitting antenna $P_{t}$ is given by the Friis equation,

$$
P_{r}=P_{t}+G_{t}+G_{r}+20 \log _{10}\left(\frac{\lambda}{4 \pi R}\right)^{2}
$$

where $G_{t}$ and $G_{r}$ are the antenna gains of the transmitting and receiving antennas, respectively; $\lambda$ is the wavelength; and $R$ is the distance. Therefore, the gain of an antenna is a measure of its overall effectiveness in transducing an incident electric field across it into a usable electrical RF signal from a particular direction. Typical gains are listed for currently available linear antennas in Table 2.

In general, there is a tradeoff between the gain and angular beam width, which means that a higher gain antenna has narrower angular beams. The choice of antenna depends on the relative position between the power source and power receiver. When harvesting power from a TV tower, high-gain antennas such as log periodic antennas are more useful, as seen in [9]. However, the electromagnetic field leaked from microwave ovens shows a significant spatial and temporal change in front of the door. Thus, antennas with wider beam widths, such as monopole and dipole antennas, are preferable.

\begin{tabular}{|c|c|c|}
\hline Antenna Type & Gain (dBi) & Bandwidth \\
\hline \hline Dipole & 1.76 & High \\
Monopole & 3 & High \\
Microstrip Patch & $6-9$ & Low \\
Log Periodic & $4-9$ & Medium \\
Arrays & $6-15$ & Low \\
Horn & 20 & Low \\
Dish Antennas & 61 & Medium \\
\hline
\end{tabular}

Table 2. Gain and bandwidth of different antenna types.

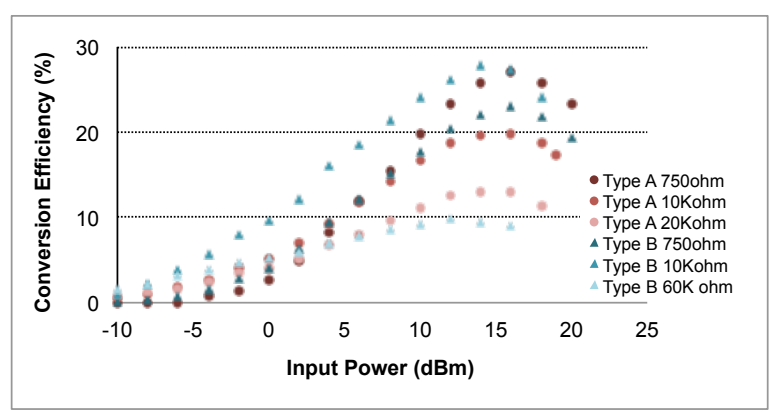

Figure 7. Conversion efficiency vs. input power level.

\section{MEASUREMENT EXPERIMENT}

\section{Objective}

The ultimate question for the feasibility of power harvesting from microwave oven leakage is how much power is harvested per day and how far the receiver can be from the oven. The power leakage from a microwave oven is not intentionally designed specifically for power harvesting. Thus, thorough characterization is necessary to exploit the power as an energy source. We took measurements in an experiment using the rectifier presented in the previous section as shown in Figure 6. The results showed that the incident power the rectifier can harvest significantly changes depending on the distance from the oven, cooking time, and food contained in the chamber.

\section{Conversion Efficiency}

The standalone conversion efficiency of the charge pump was tested. Figure 7 shows the conversion efficiency of the charge pump A and B. The charge pump was connected to a vector signal generator (Rohde \& Schwarz SMJ100A). The output of the rectifier was connected to a resistor. The output power was calculated from the voltage measured across the resistor using a digital oscilloscope. As shown in Figure 7, the conversion efficiency significantly changed depending on the input power level and load connected to the output terminal. Note that this measurement was conducted by connecting the chargepump directly to a signal generator to show the trend caused by the input power and conversion efficiency. Accurately speaking, what these figures tell is nothing but the conversion efficiency changes depending on the input power and output load. The conversion efficiency itself may change depending on the impedance of the antenna for harvesting. By improving the impedance matching, the peak conversion efficiency can be increased to up to $70 \%$. 


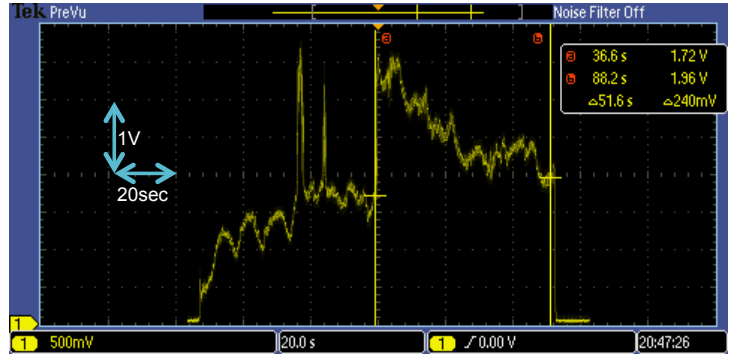

(a) Empty

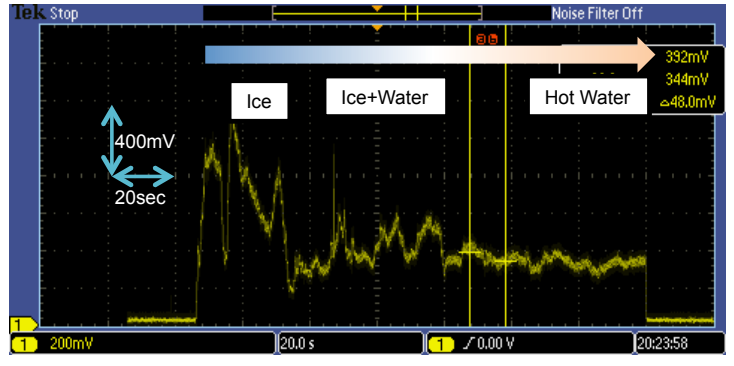

(c) Ice

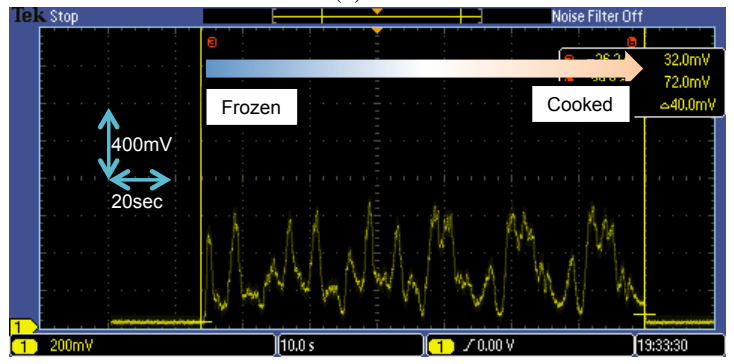

(e) Dumpling

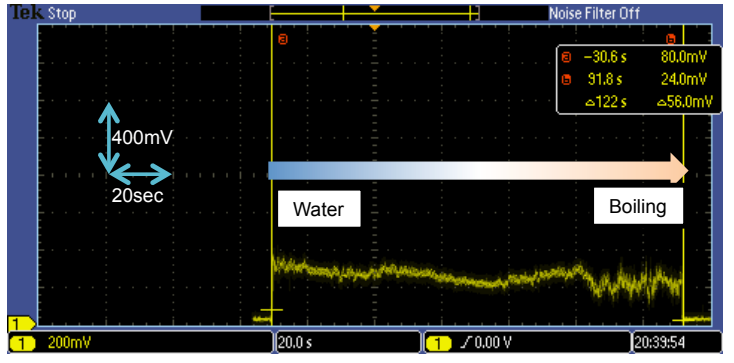

(b) Water

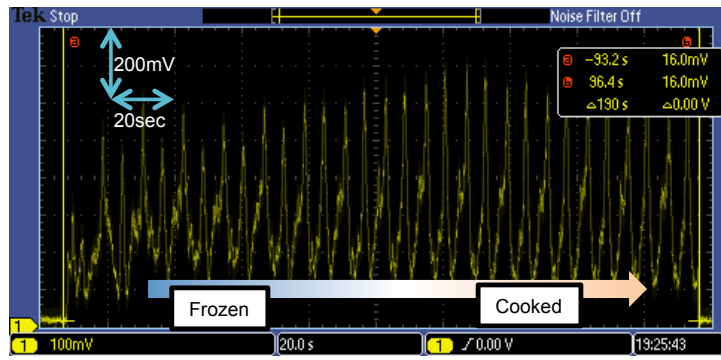

(d) Fried rice

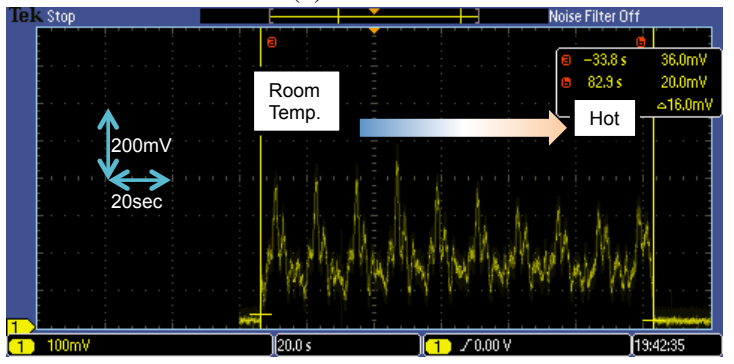

(f) Spaghett

Figure 8. Time change of output voltage and contents in the chamber.

\section{Observation of temporal variation of harvested power}

The next measurement was taken using a Type A charge pump. A dipole antenna was connected to the input of the charge pump, and a resistor of $470 \mathrm{k} \Omega$ was connected to the output. A capacitor of $1.5 \mu \mathrm{F}$ was also connected in parallel to the load resistor. The voltage across the resistor was measured using a digital oscilloscope. The antenna was located $50 \mathrm{~cm}$ away from the center from the door of the microwave oven to avoid coupling of the metal shell of the oven and antenna. The rest of the measurement was conducted using National NE-EZ2. The microwave oven was operated at a maximum power of $700 \mathrm{~W}$. During the operation, one of the following items was placed in the oven chamber: (a) empty cup, (b) $200 \mathrm{ml}$ tap water in a glass, (c) ice (200 g), (d) frozen fried rice $(180 \mathrm{~g})$, (e) frozen dumpling, and (f) cooked spaghetti $(100 \mathrm{~g})$. The results are shown in Figure 8 . These measurements results imply several important facts regarding energy harvesting from microwave oven. First, temporal variation of the output voltage is always present. The output voltage was quite stable when the power was directly fed by a signal generator during the measurement of standalone conversion efficiency. Possible causes for the large temporal variation observed during the measurement are summarized below.
(1) As seen in all cases except Figure 8 (b), a periodic "spike" was observed every 5.79 or $11.58 \mathrm{~s}$. This cycle coincided with the rotation of the turntable in the chamber. Most entryclass microwave ovens have a rotating turntable to disperse the microwave energy across the content in the chamber. The cycle of the turntable in the National NE-EZ2 was about 12 $\mathrm{s}$. The electromagnetic field generated by a magnetron traveling into the chamber is reflected when it hits a wall inside the chamber and attenuated when it hits dielectric substances such as water molecule. Each electromagnetic field directed to slightly different directions experiences a different attenuation, delay, and phase shift while traveling inside the chamber. Thus, the changing locations of objects on the turntable can result in significant constructive or destructive interference in the chamber.

(2) The output voltage varies depending on the condition of the contents in the chamber. As shown in Figure 8 (b), the voltage slightly decreased when the water in the glass started to boil $90 \mathrm{~s}$ after operation. When water began to boil, the surface of the water became ruffled. This motion of the water surface disturbed the electromagnetic field. Figure 8 (c) shows another interesting phenomenon peculiar to microwave heating. The output voltage was higher when the glass contained ice and decreased as the ice melted. The temporal variation was stable by the time all of the ice turned to wa- 


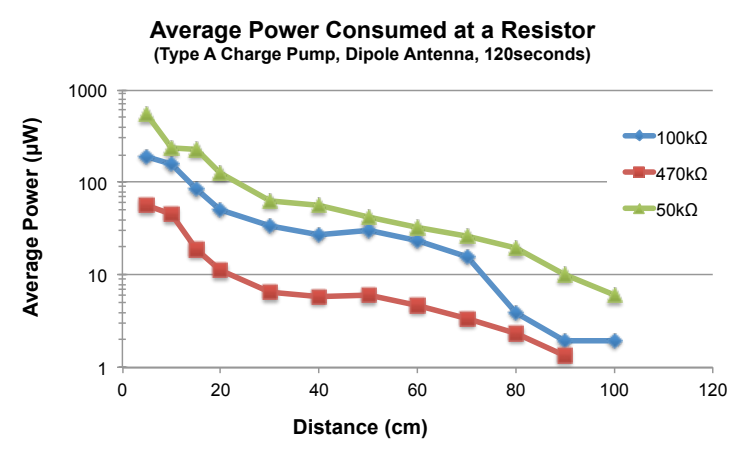

Figure 9. Average power consumption over $120 \mathrm{~s}$.

ter. The principle for how content in a microwave oven behaves is based on the microwave heating phenomenon. According to the theory of microwave heating, friction resulting from molecule alignment and migration of charged ions in rapidly alternating electromagnetic field generates heat within foods. Therefore, the efficiency of power absorption is related to the dielectric property of the substance. The power observed by the dielectric material is theoretically calculated by $P=0.556 \times 10^{-12} \cdot \varepsilon_{r} \cdot \tan \delta \cdot f \cdot E^{2}$, where $\varepsilon_{r}$ is the dielectric constant, $\tan \delta$ is the dissipation factor, $f$ is the heating frequency, and $E$ is the electrical field intensity. Here, $\varepsilon_{r}$ and $\tan \delta$ differ for each material as well as the status of the material. For example $\varepsilon_{r}$ and $\tan \delta$ of water at $20^{\circ} \mathrm{C}$ are about 80 and 0.2 respectively, while those of ice are 3 and 0.001 , respectively. Therefore, when only ice was present in the chamber, the energy was not absorbed by the ice and leaked out of the front door. When the surface of the ice was covered with a small amount of water, the water started to absorb the microwave energy, which accelerated the melting of the ice. Figure 8 (c) demonstrates a good example of the phenomenon. In case of frozen (d, e) and cooked (f) foods, the change in output voltage due to the status of change was not obvious. We believe that this is because the ice contained in the food rapidly became water vapor, which did not affect the overall absorption of microwave power in the chamber.

\section{Available Power over Time vs. Distance from Oven}

Figure 9 shows the average power consumption over $120 \mathrm{~s}$ in front of the center of the door panel. The chamber was empty. According to the measurement results, the total energy consumed by a resistor of $50 \mathrm{k} \Omega$ over $120 \mathrm{~s}$ at distances of 5,20 , 50 , and $100 \mathrm{~cm}$ were $540,126,42$, and $6 \mu \mathrm{W}$, respectively.

The harvested power decreased with increasing distance from the oven. The drop was more significant when the distance was within $20 \mathrm{~cm}$. This is because the antenna was within the near field region, where the microwave source and receiving antenna electromagnetically interfere with each other. At distances of more than $30 \mathrm{~cm}$, the energy decayed according to the Friis equation.

\section{OPERATION OF LOW-POWER DEVICES USING THE HAR- VESTED ENERGY}

Figure 10 shows the power consumption of a cooking thermometer (Tanita TT-533-GR), a humidity and temperature

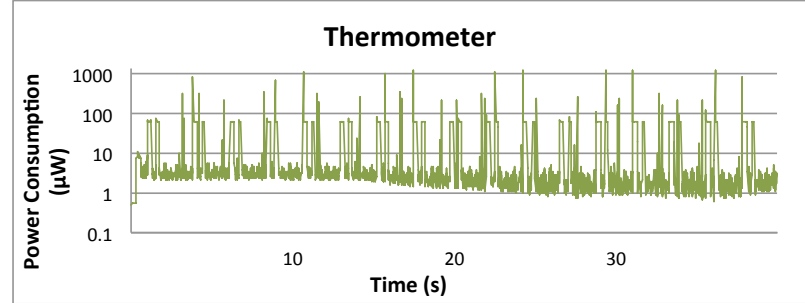

(a) Cooking thermometer (average:23.9 $\mu \mathrm{W}$ )

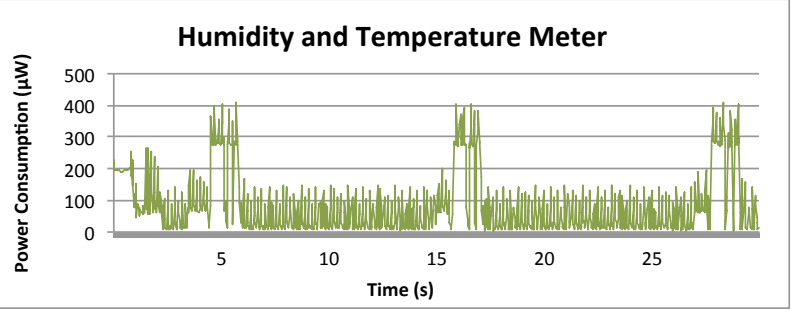

(b) Humidity and temperature meter (average: $70 \mu \mathrm{W}$ )

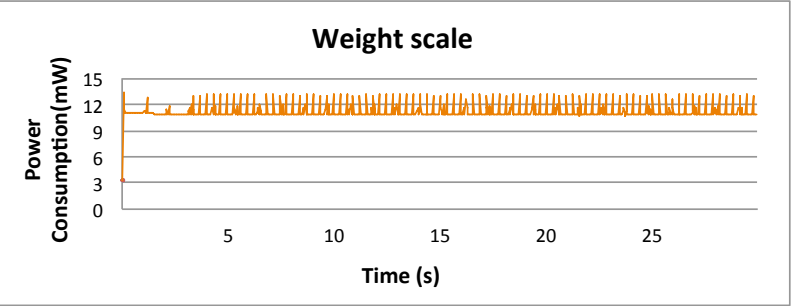

(c) Weight scale (average: $10 \mathrm{~mW}$ )

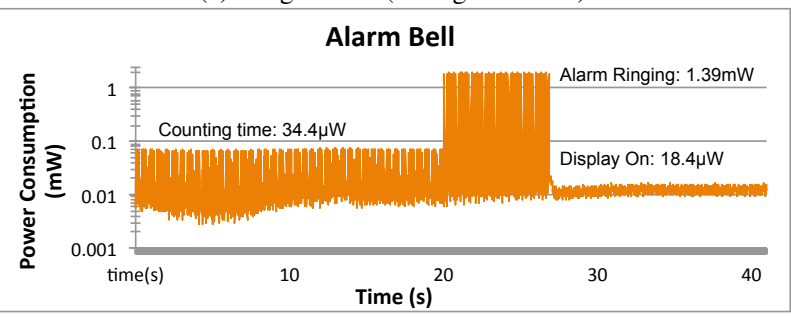

(d) Digital Kitchen timer

Figure 10. Digital kitchen devices and their power consumption.

meter (Dretec O-226 WT), a weight scale (Pearl life, D-12), and a digital kitchen timer (Tanita TD-379-BK). Each device has different power requirements. The average power consumptions of a cooking thermometer and humidity meter are 23.9 and $70 \mu \mathrm{W}$, respectively. The power consumption of the thermometer and humidity meter are almost equal to the harvested power within 15 and $60 \mathrm{~cm}$ from the microwave oven.

Note that, instead of connecting these devices at the output terminal of the charge pump, it is more desirable to connect a larger capacitor having a capacity such as $1000 \mu \mathrm{F}$ to sustain the sudden increase often found during the operation of the load device and decrease in leakage power level. A capacitor with a size of $C[\mathrm{~F}]$ can hold an energy of $\frac{1}{2} C V^{2}[\mathrm{~J}]$. A power consumption of $100 \mu \mathrm{W}$ lasting $2 \mathrm{~s}$ is equal to 200 $\mu \mathrm{J}$. If a capacity of $1000 \mu \mathrm{F}$ is connected to the charge pump and charged up to $1.7 \mathrm{~V}$, the capacitor's terminal voltage after consumption of $200 \mu \mathrm{J}$ is still $1.58 \mathrm{~V}$, which is higher than the ideal battery level of button cells such as LR44. 


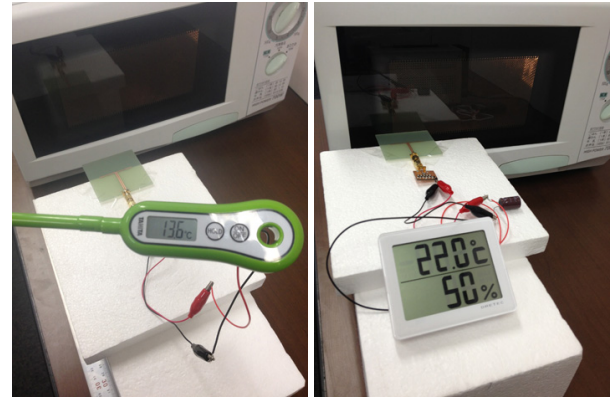

Figure 11. Kitchen devices operated by harvested energy.

Figure 11 shows those devices in operation. The weight scale and digital kitchen timer are trickier. The weight scale constantly consumes $10 \mathrm{~mW}$ from a $3 \mathrm{~V}$ power supply of CR2032. In order to operate the scale for $9 \mathrm{~s}$, an energy of $90 \mathrm{~mJ}$ is necessary. This energy is almost equal to the total amount of energy that the Type A charge pump can collect in $120 \mathrm{~s}$ from $5 \mathrm{~cm}$ in front of the door.

The overall power consumption of a digital kitchen timer is relatively small. The power consumptions when it is counting down (or up) and in an idle state are 34.4 and $18.4 \mu \mathrm{W}$, respectively. However, once it starts beeping, it consumes 1.39 $\mathrm{mW}$. Thus, when a capacitor of $1000 \mu \mathrm{F}$ holds $10 \mathrm{~mJ}$, the kitchen timer can count up about $4 \mathrm{~min}$ and $22 \mathrm{~s}(9.0 \mathrm{~mJ})$ or count down $180 \mathrm{~s}$ and beep for $2.5 \mathrm{~s}(9.7 \mathrm{~mJ})$.

Operation of such kitchen devices is actually more challenging than operation of wireless sensor network nodes. For instance, Texas Instruments eZ430-RF2500 sensor node contains an MSP430F2274 micro controller, and CC2500 transceiver with SimpliciTI protocol stack and a temperature sensor. This sensor can sense temperature data and send it to a remote sink node using only $200 \mu \mathrm{J}$ and current consumption in sleep mode is about $1 \mu \mathrm{A}$. Hence, as long as a sensor node wakes up intermittently, the sensor node can do sensing once in half an hour for 2.5 hours using $10 \mathrm{~mJ}$ of energy.

\section{Accumulation to capacitor}

As explained previously, the conversion efficiency of the charge pump changes depending on the output load resistance. We also measured the total accumulated power over $120 \mathrm{~s}$ using a Type A rectifier and dipole antenna (Figure 12).

The maximum energy stored at the capacitor was $9.98 \mathrm{~mJ}$ at $5 \mathrm{~cm}$ away from the door. The measurement results showed that the total amount of energy stored by the capacitor can be decreased down to $15 \%$ of the optimal power revealed in Figure 9.

\section{DISCUSSION}

The measurement results can be summarized as follows. All of the leakage must be suppressed to less than $5 \mathrm{~mW} / \mathrm{cm}^{2}$ according to regulations. In reality, the leakage level was a maximum of $500 \mu \mathrm{W} / \mathrm{cm}^{2}$. The instantaneous power levels received when a dipole antenna was used was about $0 \mathrm{dBm}$ $(1 \mathrm{~mW})$. The maximum average power the resistor could consume was $88.7 \mathrm{~mJ}$, while a capacitor of $1000 \mu \mathrm{F}$ could store $9.98 \mathrm{~mJ}$ after $2 \mathrm{~min}$ of operation (Figure 13).

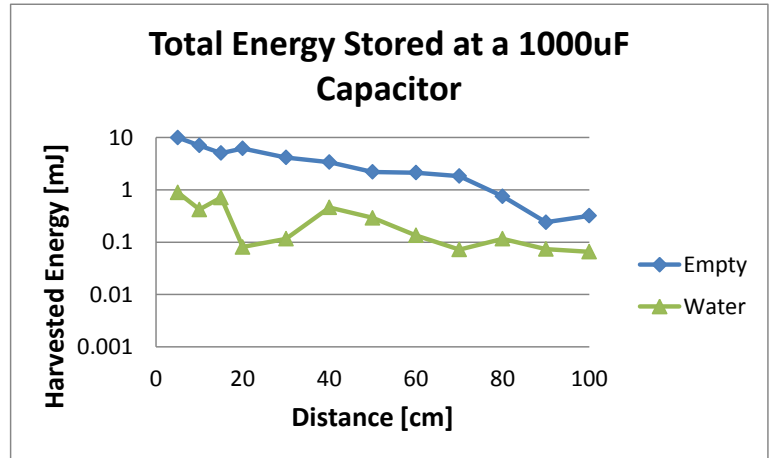

Figure 12. Total energy accumulated over 120s.

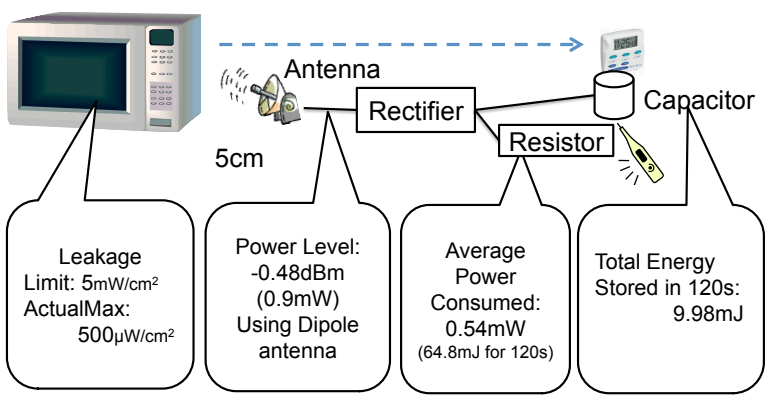

Figure 13. Summary of available energy.

\section{Effect on antenna type on efficiency}

Based on a calculation, the efficiency of converting the energy received by antenna into a DC energy is 0.54 $\mathrm{mW} / 0.9 \mathrm{~mW}=60 \%$. However, we would note these numbers include a lot of uncertainty. The received channel power of $-0.48 \mathrm{dBm}$ was instantaneous value measured at a spectrum analyzer whose input impedance is $50 \Omega$. Therefore the amount may vary depending on the impedance of the antenna used. Especially, the charge pump is designed to match with a dipole antenna. Moreover the interference between antenna and microwave oven causes impedance change in antenna in such a close distance as $5 \mathrm{~cm}$. Most critical reason is the electrical field strength constantly changes as seen in Figure 3. In general, antenna's sensitivity changes depending on the frequency.

We have conducted one more experiment about the difference of the chagepump, antenna, microwave oven, and content in the oven. The measurement was conducted using a microwave oven C (Sharp, R55TS). Type-A rectifier is connected to a dipole antenna and Type-B rectifier is connected to a high gain antenna (TRENDNET TEW-AO14D, $14 \mathrm{dBi}$ ). The microwave oven are operated for two minutes with and without a glass of water in the chamber. See Figure 14 for the result. Though the microwave oven was different, amount of energy stored at a capacitor after $120 \mathrm{~s}$ at $5 \mathrm{~cm}$ away from the door was $6.85 \mathrm{~mJ}$ (Type-B, Empty), $6.09 \mathrm{~mJ}$ (Type-A, Empty), $5.36 \mathrm{~mJ}$ (Type-B, Water) and $3.38 \mathrm{~mJ}$ (Type-A, Water), respectively. In this case, the energy decrease caused by presence of water is minor than National. It is also remarkable that use of high-gain antenna can significantly increase the harvested power at longer distances. However, it 


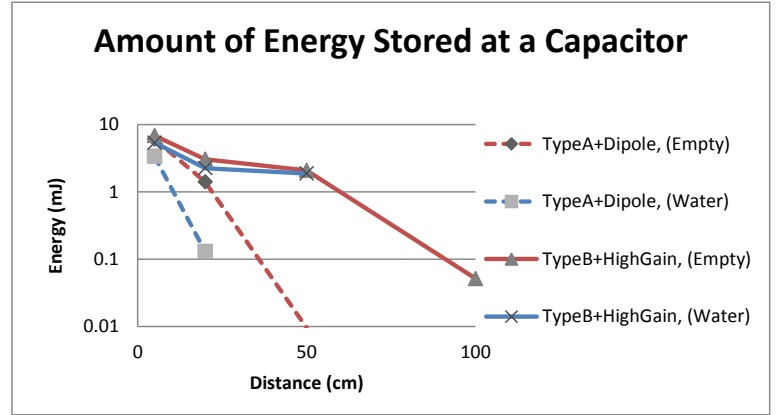

Figure 14. Amount of energy using different antenna.

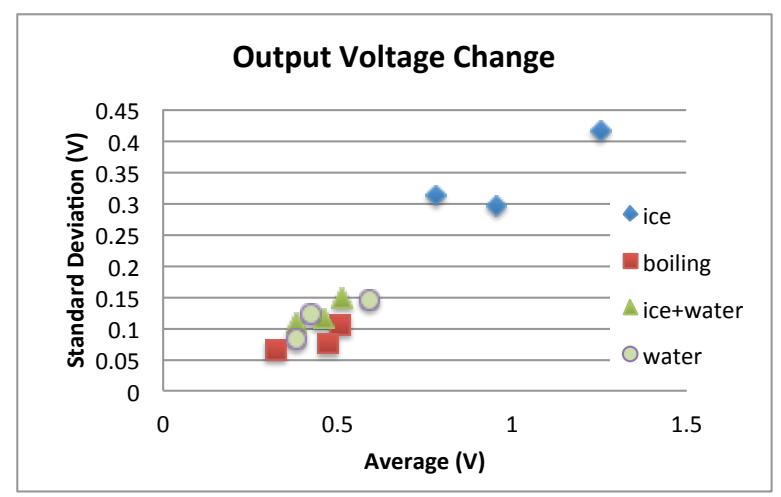

Figure 15. Variation of amount of power by status of food.

also shows high gain antenna doesn't contribute to the increase of the harvested power much compared to a dipole antenna when it is located close to the microwave oven. One of the reason is high gain antenna is more susceptible to interference with the body of microwave oven. Even though there is always much uncertainty when measuring electromagnetic field under practical settings, the measurement settings we chosen were not too optimistic, we believe. In fact, we could further improve the accumulated energy from leakage of microwave ovens especially by using more sophisticated impedance matching. The amount of energy stored in the capacitor after 2 min of operation was only $15 \%$ of the ideal case. This is because the conversion efficiency changes depending on the load resistance. If the energy being received and used for a load is already known, power management circuits, such as those in [9], will improve this accumulation drastically. When the duty cycle of the microcontroller in the device can be explicitly controlled, the optimal duty cycle can be determined [13]. In addition, when an array of rectennas can also be used to double the available power[4].

The large temporal variation during the operation of microwave oven shown in Figure 8 also implies possibility of inference about contents and its cooking state in the microwave oven chamber. According to the experiment result, average and standard deviation of an output voltage when a glass of water are different depending on the state when an ice is heated in the oven as seen in Figure 15. This fluctuation is caused by the change in electromagnetic property of the food (permittivity and loss tangent). The pattern also changes depending on the shape of the content (e.g. ice v.s. water). Since the amount of the content also affects the absolute leakage amount, there still needs thorough investigation under fully controlled conditions on many parameters. However this experiment result imply that external sensing devices may detect what's cooked and how they are cooked. Such information will be essential information to track daily eating behavior of patients afflicted with lifestyle-related diseases. Commercially available high-end microwave ovens can identify the contents and its cooking state by exploiting various sensing technologies including a weight sensor, infrared temperature sensors and a humidity sensor. Such sensing information is used to adjust the cooking time and output power depending on the content and its cooking state but such information is cannot be exported to other devices.

\section{CONCLUSION AND FUTURE WORK}

We demonstrated the feasibility of harvesting several hundreds of microwatts of electricity from the leaked electromagnetic field of a microwave oven. The instantaneous power levels received when a dipole antenna was used was about $0.9 \mathrm{~mW}$. The maximum average power a load resistor could consume was $64.8 \mathrm{~mJ}$, while a capacitor of $1000 \mu \mathrm{F}$ could store $9.98 \mathrm{~mJ}$ after $2 \mathrm{~min}$ of operation. The amount of harvested power was similar even though a combination of charge pump, antenna and a microwave oven was different.

The energy accumulated over 2 min was found to be sufficient for the operation of some of low-power kitchen tools for a few minutes and operate wireless sensor node for 2.5 hours. The amount of energy stored in the capacitor after $2 \mathrm{~min}$ of operation was only $15 \%$ of the ideal case. We could further improve the accumulated energy from leakage of microwave ovens by using more sophisticated impedance matching and power management methods.

\section{ACKNOWLEDGMENTS}

We would like to thank reviewers who gave us a lot of useful technical suggestions and comments.

This research was supported in part by the Industrial Technology Research Grant Program, 2009, of the New Energy and Industrial Technology Development Organization (NEDO) of Japan and JSPS KAKENHI Grant Numbers 22680004.

\section{REFERENCES}

1. Performance Standards for Microwave and Radio Frequency Emmiting Products. Code of Federal Regulations Title 21, (2012).

2. Brown, W. An experimental low power density rectenna. In 1991 IEEE MTT-S International Microwave Symposium Digest, 197-200.

3. De Vita, G., and Iannaccon, G. Design criteria for the RF section of UHF and microwave passive RFID transponders. IEEE Transactions on Microwave Theory and Techniques 53, 9 (Sept. 2005), 2978-2990.

4. Hagerty, J., Helmbrecht, F., McCalpin, W., Zane, R., and Popovic, Z. Recycling Ambient Microwave Energy With Broad-Band Rectenna Arrays. IEEE Transactions on Microwave Theory and Techniques 52, 3 (Mar. 2004), 1014-1024. 
5. Merabet, B., Cirio, L., Takhedmit, H., Costa, F., Vollaire, C., Allard, B., and Picon, O. Low-cost converter for harvesting of microwave electromagnetic energy. In Proc. IEEE Energy Conversion Congress and Exposition, 2009, IEEE (2009), 2592-2599.

6. Monti, G., Congedo, F., Donno, D., and Tarricone, L. Monopole-based rectenna for microwave energy harvesting of UHF RFID systems. Progress In Electromagnetics Research C 31 (2012), 109-121.

7. Nassar, M., Lin, X. E., and Evans, B. L. Stochastic Modeling of Microwave Oven Interference in WLANs. In 2011 IEEE International Conference on Communications (ICC), IEEE (June 2011), 1-6.

8. Nishimoto, H., Kawahara, Y., and Asami, T. Prototype implementation of ambient RF energy harvesting wireless sensor networks. In 2010 IEEE Sensors, IEEE (Nov. 2010), 1282-1287.

9. Parks, A., Sample, A., Zhao, Y., and Smith, J. R. A Wireless Sensing Platform Utilizing Ambient RF Energy. In IEEE Topical Meeting on Wireless Sensors and Sensor Networks (WiSNET) (Austin, TX, 2013).

10. Patel, S., Stuntebeck, E., and Robertson, T. PL-Tags: Detecting Batteryless Tags through the Power Lines in a
Building. In Pervasive Computing, vol. 5538 of Lecture Notes in Computer Science, Springer Berlin Heidelberg (2009), 256-273.

11. Sample, A., and Smith, J. R. Experimental results with two wireless power transfer systems. In 2009 IEEE Radio and Wireless Symposium, IEEE (Jan. 2009), 16-18.

12. Sample, A., Yeager, D., Powledge, P., and Smith, J. Design of a Passively-Powered, Programmable Sensing Platform for UHF RFID Systems. In 2007 IEEE International Conference on RFID, IEEE (2007), 149-156.

13. Shigeta, R., Sasaki, T., Quan, D., Kawahara, Y., Vyas, R., Tentzeris, M., and Asami, T. Ambient-rf-energy-harvesting sensor device with capacitor-leakage-aware duty cycle control. IEEE Sensors Journal PP, 99 (2013), 1-10.

14. Vyas, R., Cook, B., Kawahara, Y., and Tentzeris, M. E-wehp: A batteryless embedded sensor-platform wirelessly powered from ambient digital-tv signals. IEEE Transactions on Microwave Theory and Techniques 61, 6 (2013), 2491-2505. 